\title{
New vistas in charm production
}

\author{
Antoni Szczurek ${ }^{1,2, *}$ \\ ${ }^{1}$ Institute of Nuclear Physics PAN, Kraków, Poland \\ ${ }^{2}$ Rzeszów University, Rzeszów, Poland
}

\begin{abstract}
We discuss some new aspects of charm production trigerred by recent observations of the LHCb collaboration. The LHCb collaboration measured small asymmetries in production of $D^{+} D^{-}$mesons as well as $D_{s}^{+} D_{s}^{-}$mesons. Is this related to initial quark/antiquark asymmetries in the proton? Here we discuss a scenario in which unfavored fragmentations $q / \bar{q} \rightarrow D$ and $s / \bar{s} \rightarrow D_{s}$ are responsible for the asymmetries. We fix the strength of such fragmentations - transition probabilities, by adjusting to the size of the LHCb asymmetries. This has consequences for production of $D$ mesons in forward directions (large $\left.x_{F}\right)$ as well as at low energies. Large asymmetries are predicted then in these regions. We present here some of our predictions. Consequences for highenergy neutrino production in the atmosphere are discussed and quantified. The production of $\Lambda_{c}$ baryon at the LHC is disussed. Large deviations from the independent-parton fragmentation picture are found.
\end{abstract}

\section{Introduction}

It is usually assumed that the $c / \bar{c} \rightarrow D$ fragmentation is responible for production of charmed mesons. In leading order $g g \rightarrow c \bar{c}$ is dominant partonic subprocess. The contribution of $q \bar{q} \rightarrow$ $c \bar{c}$ is usually much smaller. The leading-order production of charm is by far insufficient to describe experimental distributions of $D$ mesons in rapidity and transverse momentum. The NLO calculation is needed to describe experimental data. An alternative is the $k_{t}$-factorization approach which gives resonable description of $D$ meson single particle distributions [1]. It allows to describe even some correlation observables [2]. Usually the Peterson fragmentation functions [3] are used for $c \bar{c} \rightarrow D$ fragmentations.

Recently the LHCb collaboration observed an intriguing asymmetries for $D^{+} D^{-}$[4] and $D_{s}^{+} D_{s}^{-}[5]$ production. The question arises what is origin of such asymmetries. In general, there can be a few reasons such as electroweak corrections, higher-order pQCD effects. The electroweak corrections should be important rather at large transverse momenta. The LHCb collaboration measured the asymmetries at rather small transverse momenta where statistics is enough to pin down the small asymmetry effect. In Fig.1 we show for ilustration distribution of partons obtained in LO collinear approach. Furthermore the distribution of light quarks and even antiquarks is much larger than the distribution of $c / \bar{c}$ quarks/antiquarks produced in gluon-gluon fusion process. The distribution of light quarks is much larger than distribution of corresponding antiquarks. All this suggests that a nonzero subleading fragmentation $d \rightarrow D^{-}$and $\bar{d} \rightarrow D^{+}$would produce an asymmetry when added to the dominant

\footnotetext{
*e-mail: ifj.edu.pl
} 
$c / \bar{c} \rightarrow D$ fragmentation. For $D_{s}$ meson production asymmetry the situation is more subtle as far as subleading fragmentation is considered. Here we have $\bar{s} \rightarrow D^{+}$and $s \rightarrow D^{-}$subleading fragmentations. If $s(x)=\bar{s}(x)$ then of course the asymmetry is zero. There are no deep reasons to assume $s(x)=\bar{s}(x)$. Actually the nonperturbative effects of the strange meson cloud lead to $s(x) \neq \bar{s}(x)$ (see e.g.[7]). Also some fits of parton distributions allow for different distributions of $s$ and $\bar{s}$ partons [8].
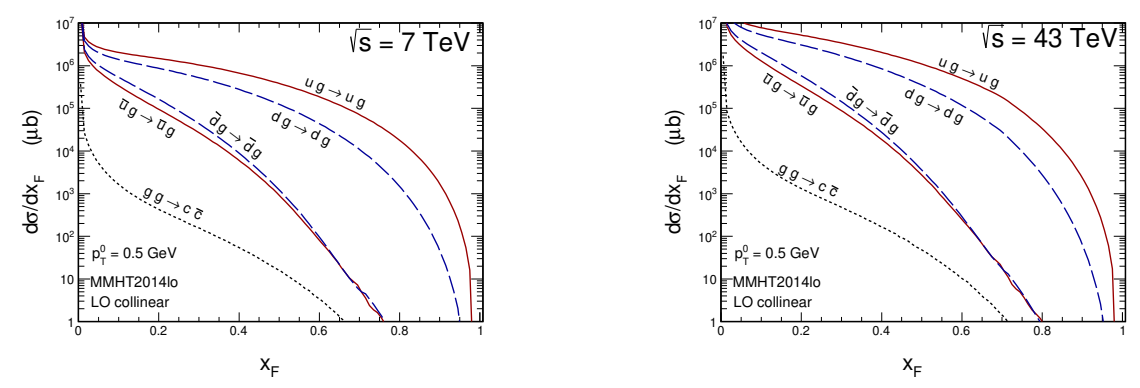

Figure 1. Quark and antiquark distributions in Feynman $x_{F}$ for $\sqrt{s}=7 \mathrm{TeV}$ (left panel) and $\sqrt{s}=43$ $\mathrm{TeV}$ (right panel) corresponding to $E_{\mathrm{lab}}(p)=10^{9} \mathrm{GeV}$. This calculation was performed within collinearfactorization approach with somewhat arbitrary regularization parameter $p_{T}^{0}=0.5 \mathrm{GeV}[6]$.

\section{Cross sections, production asymmetry and subleading fragmentations}

Let us discuss first the dominant at the LHC contribution - the gluon-gluon fusion. The multi-diferential cross section for $c \bar{c}$ productions can be then calculated as:

$$
\begin{aligned}
\frac{d \sigma(p p \rightarrow c \bar{c} X)}{d y_{1} d y_{2} d^{2} p_{1, t} d^{2} p_{2, t}}= & \int \frac{d^{2} k_{1, t}}{\pi} \frac{d^{2} k_{2, t}}{\pi} \frac{1}{16 \pi^{2}\left(x_{1} x_{2} s\right)^{2}} \overline{\left|\mathcal{M}_{g^{*} g^{*} \rightarrow c \bar{c}}^{\text {off }}\right|^{2}} \\
& \times \delta^{2}\left(\vec{k}_{1, t}+\vec{k}_{2, t}-\vec{p}_{1, t}-\vec{p}_{2, t}\right) \mathcal{F}_{g}\left(x_{1}, k_{1, t}^{2}\right) \mathcal{F}_{g}\left(x_{2}, k_{2, t}^{2}\right),
\end{aligned}
$$

where $\mathcal{F}_{g}\left(x_{1}, k_{1, t}^{2}\right)$ and $\mathcal{F}_{g}\left(x_{2}, k_{2, t}^{2}\right)$ are the gluon uPDFs for both colliding hadrons and $\mathcal{M}_{g^{*} g^{*} \rightarrow c \bar{c}}^{\text {off-shell }}$ is the off-shell matrix element for the hard subprocess. First the distribution in rapidity and transverse momentum of $c$ or $\bar{c}$ are obtained (inclusive cross section). The cross section for $D$ meson can be obtained then as a convolution of the partonic cross section for $g^{*} g^{*} \rightarrow c \bar{c}$ and the $c / \bar{c} \rightarrow D$ fragmentation functions. The Peterson fragmentation function [3] with $\epsilon$ parameter adjusted to experimental data.

In the studies presented here we include also $u, \bar{u}, d, \bar{d} \rightarrow D^{i}$ parton fragmentation to $D$ mesons. We include only fragmentations of quarks/antiquarks that are constituents of the $D$ meson. We assume the following symmetry relation:

$$
D_{d \rightarrow D^{-}}(z)=D_{\bar{d} \rightarrow D^{+}}(z)=D^{(0)}(z)
$$

Similar flavor symmetry relations hold for fragmentation of $u$ and $\bar{u}$ to $D^{0}$ and $\bar{D}^{0}$ mesons. However $D_{q \rightarrow D^{0}}(z) \neq D_{q \rightarrow D^{+}}(z)$, which is caused by the contributions from decays of vector $D^{*}$ mesons. Furthermore we assume for doubly suppressed fragmentations:

$$
D_{\bar{u} \rightarrow D^{ \pm}}(z)=D_{u \rightarrow D^{ \pm}}(z)=0 .
$$


The fragmentation functions at sufficiently large scales undergo DGLAP evolution equations. Since in the presented here analysis we are interested in small transverse momenta (small scales for DGLAP evolution) we can just use rather the initial conditions for the evolution, which are for the subleading fragmentation rather poorly known.

We parametrize the unfavoured fragmentation functions as:

$$
D_{q_{f} \rightarrow D}(z)=A_{\alpha}(1-z)^{\alpha} .
$$

Instead of fixing the uknown $A_{\alpha}$ we will operate rather with the fragmentation probabilities:

$$
P_{q_{f} \rightarrow D}=\int d z A_{\alpha}(1-z)^{\alpha}
$$

and calculate corresponding $A_{\alpha}$ for a fixed $P_{q \rightarrow D}$ and $\alpha$. Therefore in our effective approach we have only two free parameters.

Another simple option we considered in [6] is:

$$
D_{q_{f} \rightarrow D}(z)=P_{q_{f} \rightarrow D} \cdot D_{\text {Peterson }}(1-z) .
$$

Then again $P_{q_{f} \rightarrow D}$ would be the only free parameter.

The flavour asymmetry in production of $D$ mesons is defined as:

$$
A_{D^{+} / D^{-}}(\xi)=\frac{\frac{d \sigma_{D^{-}}}{d \xi}(\xi)-\frac{d \sigma_{D^{+}}}{d \xi}(\xi)}{\frac{d \sigma_{D^{-}}}{d \xi}(\xi)+\frac{d \sigma_{D^{+}}}{d \xi}(\xi)}
$$

where $\xi=x_{F}, y, p_{T},\left(y, p_{T}\right)$.

For $D_{s}$ mesons we define the production asymmetry as:

$$
A_{D_{s}^{+} / D_{s}^{-}}(\xi)=\frac{\frac{d \sigma\left(D_{s}^{+}\right)}{d \xi}(\xi)-\frac{d \sigma\left(D_{s}^{-}\right)}{d \xi}(\xi)}{\frac{d \sigma\left(D_{s}^{+}\right)}{d \xi}(\xi)+\frac{d \sigma\left(D_{s}^{-}\right)}{d \xi}(\xi)} .
$$

The production of $D_{s}$ mesons is interesting in the context of the fact that $D_{s}$ mesons are the main source of $\tau$-neutrinos:

$$
\begin{aligned}
& D_{s}^{+} \rightarrow \tau^{+}+v_{\tau} . \\
& D_{s}^{-} \rightarrow \tau^{-}+\bar{v}_{\tau}
\end{aligned}
$$

and in addition:

$$
\begin{aligned}
& \tau^{+} \rightarrow \bar{v}_{\tau}+X, \\
& \tau^{-} \rightarrow v_{\tau}+X
\end{aligned}
$$

Both emissions should be included in final evalution of $\tau$-(anti)neutrinos.

Finally in this presentation we consider production of $\Lambda_{c}$ baryons. Whether the independent parton fragmentation works for $\Lambda_{c}$ baryons was discussed in [14]. In such an approach the cross section can be written as:

$$
\left.\frac{d \sigma(p p \rightarrow h X)}{d y_{h} d^{2} p_{t, h}} \approx \int_{0}^{1} \frac{d z}{z^{2}} D_{c \rightarrow h}(z) \frac{d \sigma(p p \rightarrow c X)}{d y_{c} d^{2} p_{t, c}}\right|_{\substack{y_{c}=y_{h} \\ p_{t, c}=y_{t, h} / z}},
$$

where $p_{t, c}=\frac{p_{t, h}}{z}$ and $z$ is the fraction of longitudinal momentum of charm quark $c$ carried by a hadron $h=D, \Lambda_{c}$. A typical approximation in this formalism assumes $y_{h}=y_{c}$. 


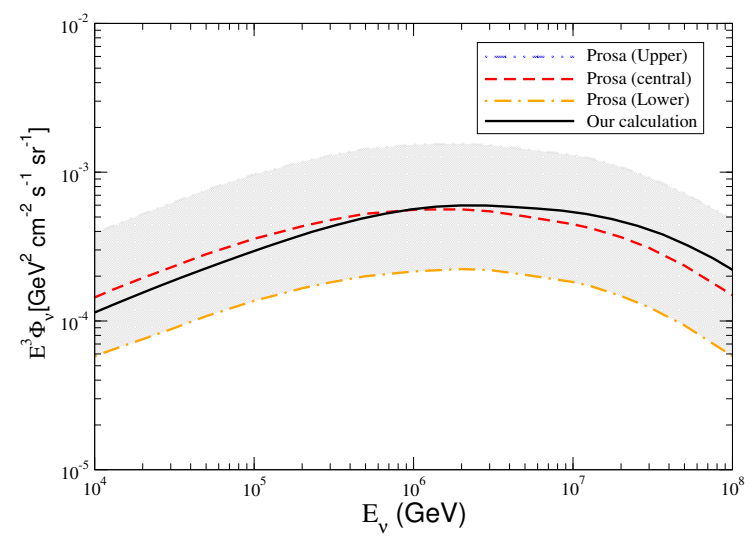

Figure 2. Comparison of our predictions for the prompt neutrino flux and the Prosa results.

\section{Results}

In this section we will show our results for (anti)neutrino production, cross sections for $D^{+} D^{-}$production and $D^{+} D^{-}$and $D_{s}^{+} D_{s}^{-}$asymmetries as well as a possible consequences for $\tau$ (anti)neutrino production and finally for $\Lambda_{c}$ baryon production.

\subsection{Neutrino production in the atmosphere}

We start from showing our best (optimal) result for neutrino flux relevant for the IceCube experiment. In Fig. 2 we show our predictions obtained for calculating cross section in the $k_{t}$-factorization approach with the KMR unintegrated gluon distributions. Such an approach effectively includes higher-order corrections as was discussed in the literature. Our result well coincides with the PROSA results within their uncertainty band.

The flux here was calculated within the $Z$-moment method [9]. In such a calculation $\frac{d \sigma}{d x_{F}}\left(x_{F}, \sqrt{s}\right)$ for production of $D$ mesons is a crucial input.

Which energies of proton-proton scattering are responsible for the production of highenergy neutrinos at IceCube? In Fig. 3 we show how the upper cut on center-of-mass energy influences the flux of high-energy neutrinos in the atmosphere. For energies $E_{v}>10^{8} \mathrm{GeV}$, the collision energies larger than those measured at the LHC enter the calculation. So predictions are based on extrapolation to unexplored yet region.

What are typical Feynman $x_{F}$ values responsible for production of high-energy neutrinos is illustrated in Fig.4. Rather large values are important. Such a region is unfortunately not covered by the LHC detectors. Even (often called) forward LHCb detector is limited to $x_{F}<$ 0.1 .

In Fig.5 we show our predictions for the flux of high-energy neutrinos. This result was obtained within $k_{t}$-factorization approach. Clearly such a calculation cannot describe the measured flux of neutrinos. No subleading fragmentations were included here. There seems to be arguments that at least part of the missing yield is of astrophysical origin [10]. Can the subleading fragmentation play a role in this context? 


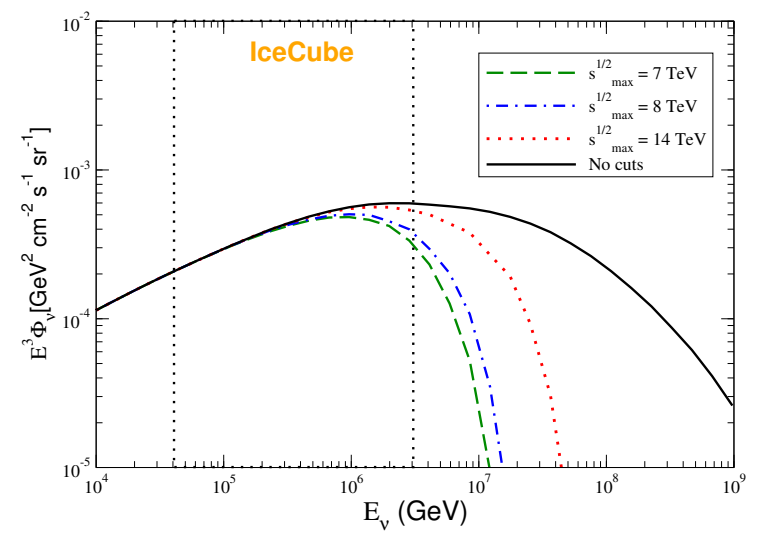

Figure 3. Impact of different cuts on the maximal center-of-mass $p p$ collision energy for the prompt neutrino flux.

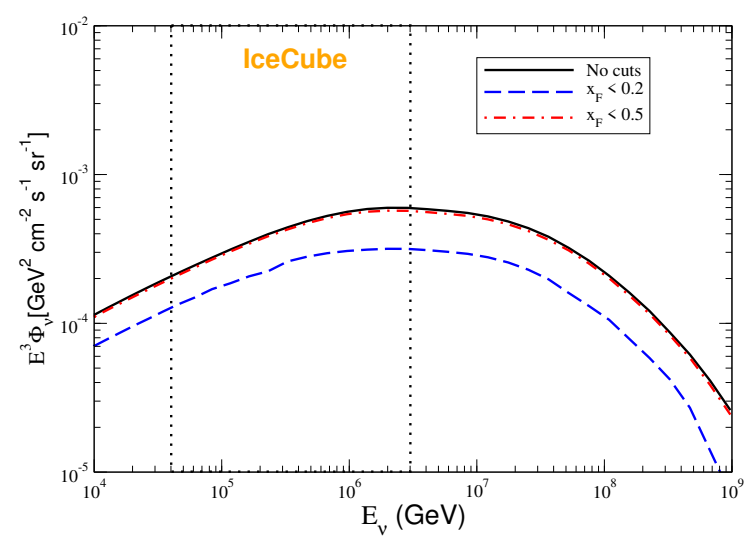

Figure 4. The effect of $x_{F}$ cuts on the prompt neutrino flux.

\subsection{LHCb asymmetries}

The $D^{+} D^{-}$asymmetries obtained by us are shown in Fig. 6 for $\sqrt{s}=7 \mathrm{TeV}$. Only one parameter, the quark/antiquark fragmentation probability, was adjusted to the LHCb data. In Ref.[6] we presented also our predictions for $\sqrt{s}=13 \mathrm{TeV}$.

Similar asymmetry for the $D_{s}^{+} D_{s}^{-}$production is shown in Fig.7. Here the error bars are even larger than for the $D^{+} D^{-}$asymmetry (see the previous figure). Again adjusting only one free parameter we can roughly reproduce the main trend of the LHCb data. Please note that our approach predicts correct sign of the asymmetry. In Ref.[12] we showed also results for $\sqrt{s}=8 \mathrm{TeV}$. 


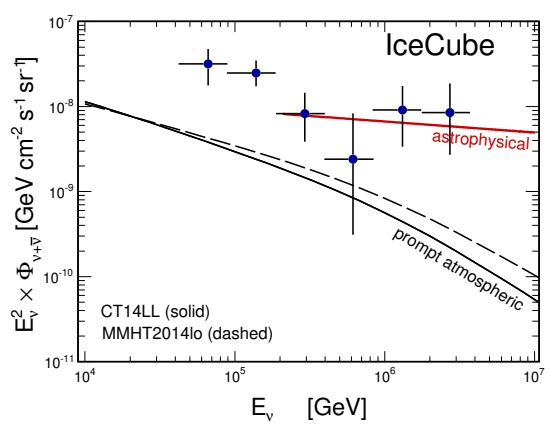

Figure 5. Comparison of predictions obtained with the CT14 and MMHT PDFs for the prompt neutrino flux. The data points are taken from IceCube analysis [11]. For comparison, a fit for the astrophysical contribution, proposed in [11] is presented as well.
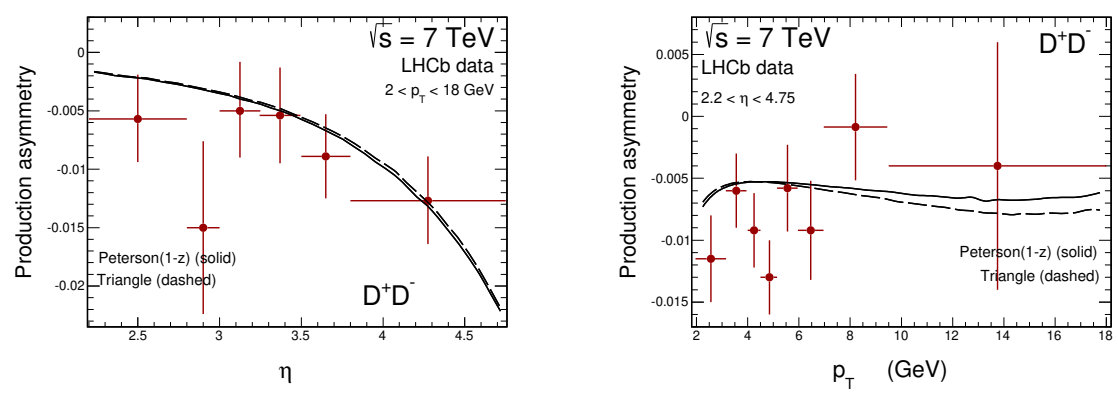

Figure 6. $A_{D^{+} / D^{-}}$production asymmetry measured by the LHCb collaboration at $\sqrt{s}=7 \mathrm{TeV}$ as a function of $D$ meson pseudorapidity (left panel) and $D$ meson transverse momentum (right panel).
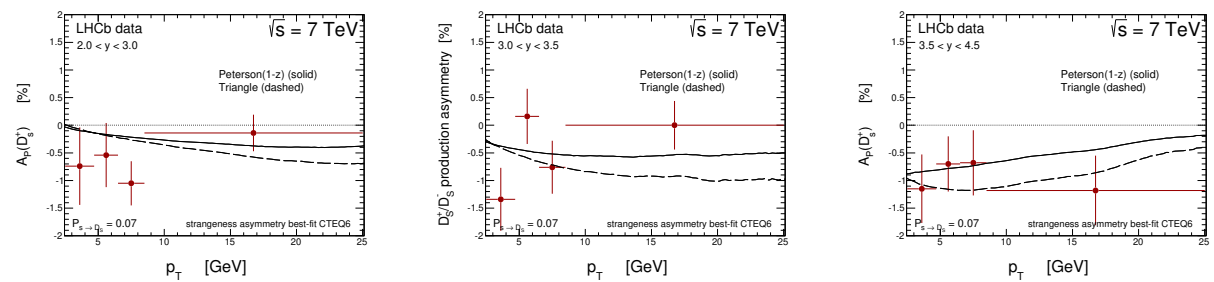

Figure 7. $D_{s}^{+} / D_{s}^{-}$asymmetry obtained by us together with the LHCb collaboration for $\sqrt{s}=7 \mathrm{TeV}$. The CTEQ6.5 parton distributions are used in this calculation.

\subsection{Asymmetries at low collision energies}

Our approach has distinct predictions at low energies. Here we show our predictions for low energies. Quite large asymmetries were found. As discussed in Ref.[6] detailed studies of the asymmetries at low energies are necessary to pin down or limit subleading fragmentation. 


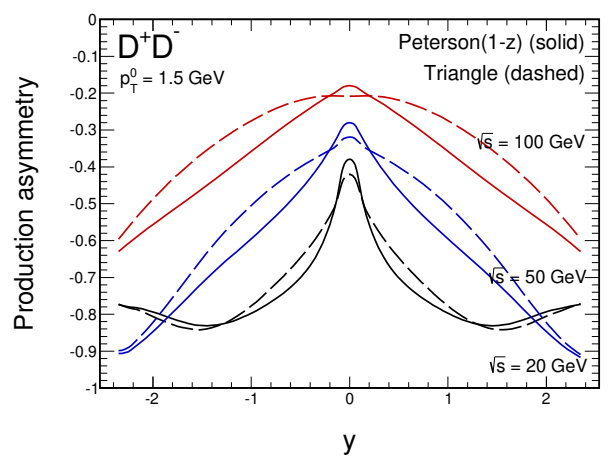

Figure 8. $A_{D^{+} D^{-}}(y)$ production asymmetry in proton-proton collisions for different center-of-mass energies $\sqrt{s}$.

\subsection{Charge-to-neutral $D$ meson ratio}

In Ref.[6] we discussed also the following ratio:

$$
R_{c / n} \equiv \frac{D^{+}+D^{-}}{D^{0}+\bar{D}^{0}}
$$

In Fig. 9 we show the ratio as a function of meson rapidity for two different energies specified in the figure. Evidently, when including subleading fragmentation, the ratio depends on collision energy and rapidity. A test of such predictions would be valuabale.
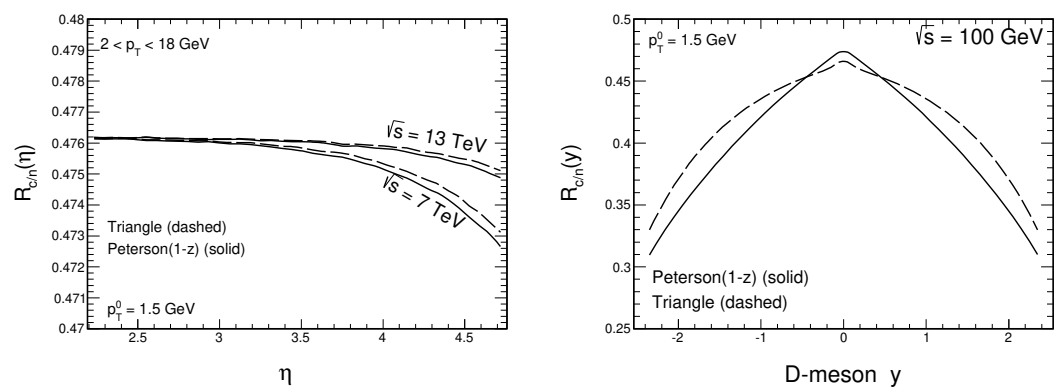

Figure 9. The $R_{c / n}$ ratio as a function of meson pseudorapidity for $\sqrt{s}=7$ and $13 \mathrm{TeV}$ for the LHCb kinematics (left panel) and as a function of meson rapidity for $\sqrt{s}=100 \mathrm{GeV}$ in the full phase-space (right panel). Only quark-gluon components (diagrams) are included here in calculating cross section for $q$ and $\bar{q}$ production.

\section{$3.5 v_{\tau}$ neutrinos and $\bar{v}_{\tau}$ antineutrinos at IceCube}

In our recent analysis we showed how the flux of $\tau$ neutrinos/antineutrinos could be modified by the subleading $s / \bar{s} \rightarrow D_{s}$ fragmentation. In Fig.10 we show the conventional flux (due to $g g \rightarrow c \bar{c}$ fusion) and that of the subleading fragmentation (left panel) as well as the corresponding ratio (right panel). The sizeable enhancement of the neutrino flux is not excluded in the moment. 

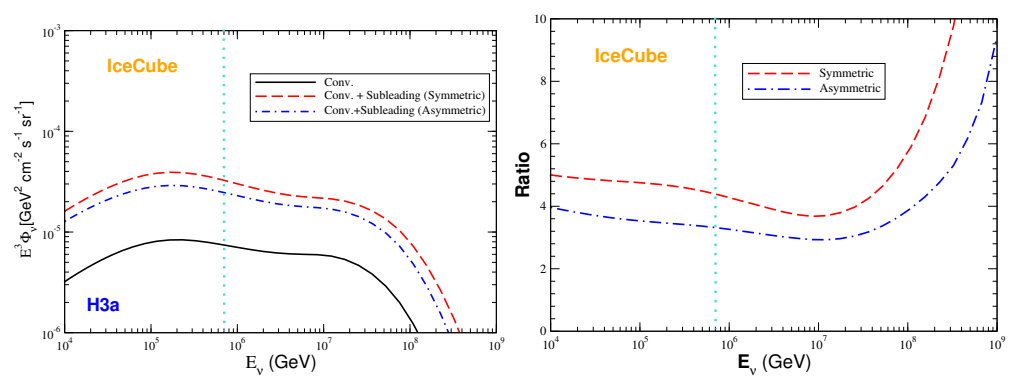

Figure 10. Our predictions for the flux of $\tau$ neutrinos (left panel) and the suggested enhancement factor with respect to the traditional $c \bar{c} \rightarrow D_{s}$ component (right panel).

\section{6 $\Lambda_{c}$ production}

In Fig. 11 we show our description of $D$ meson transverse momenta. In this calculation $y_{D}=$ $y_{c}$ was assumed. This is a standard technical prescription for $c / \bar{c} \rightarrow D$ meson production in pp collisions.
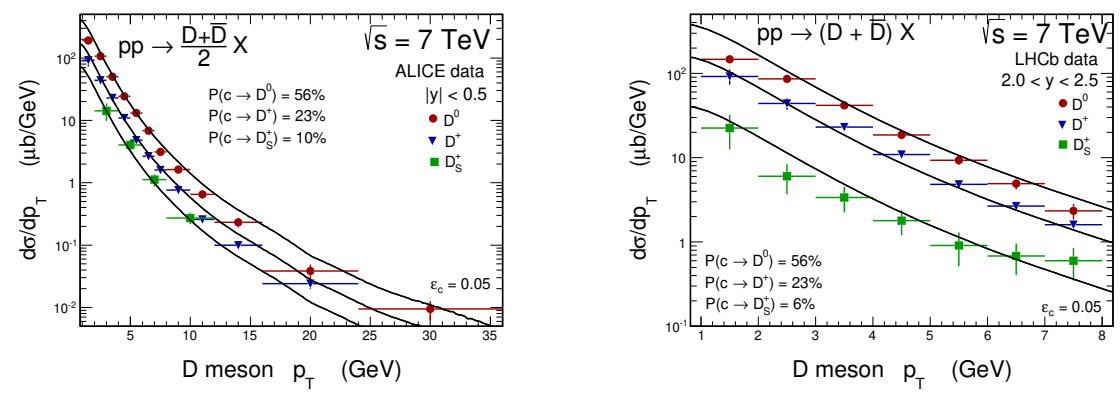

Figure 11. Transverse momentum distribution of $D$ mesons for $\sqrt{s}=7 \mathrm{TeV}$ for ALICE (left panel) and $\mathrm{LHCb}$ (right panel).

In Fig.12 we show similar results for $\Lambda_{c}$ production. We have shown our results for different $c / \bar{c} \rightarrow \Lambda_{c}$ transition probabilities. Values of the transition probability smaller than $10 \%$ were obtained from $e^{+} e^{-}$collisions. The new LHC data require much larger transition probabilities. This is especially true for the ALICE (midrapidity) data, where a value close to $20 \%$ is needed. Does it signal a new mechanism?

In Fig. 13 we show the ratio of cross section for $\Lambda_{c}$ to the cross section for $D^{0}$. This once more shows a problem of independent-parton fragmentation picture, especially at midrapidities.

In Ref.[14] we studied other options such as emissions with the assumption $\eta_{\Lambda_{c}}=\eta_{c}$ (pseudorapidities) as well as possible feed down from highly excited charmed baryons. Some small improvements, especially for the ratio, are possible but the main disagreement with independent parton fragmentation picture stays. Perhaps this could be explained in terms of a recombination model. This requires further studies and modeling of such processes. 

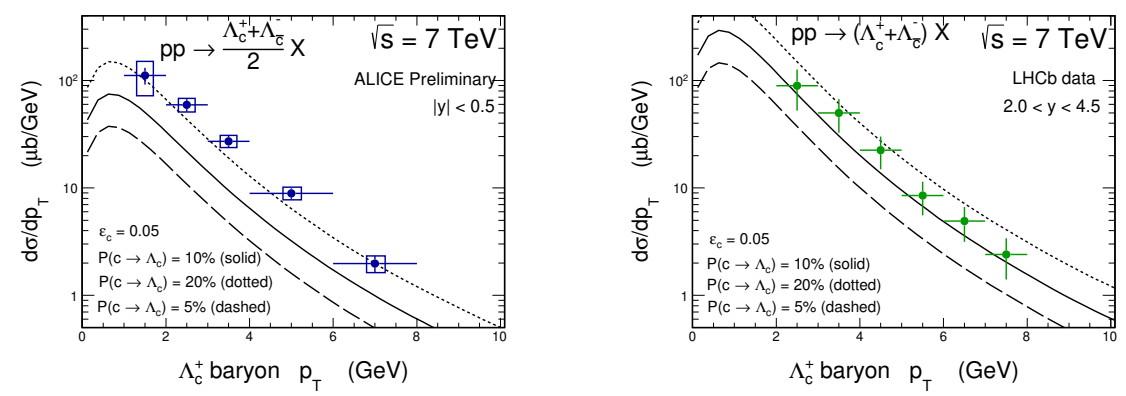

Figure 12. Transverse momentum distribution of $\Lambda_{c}$ baryon for $\sqrt{s}=7 \mathrm{TeV}$ for ALICE (left panel) and $\mathrm{LHCb}$ (right panel).
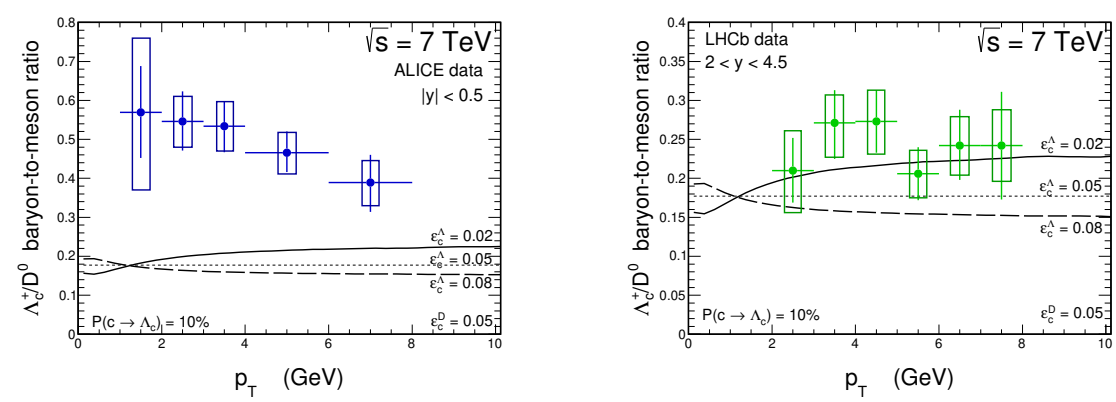

Figure 13. Transverse momentum dependence of the $\Lambda_{c} / D^{0}$ baryon-to-meson ratio for ALICE (left) and $\mathrm{LHCb}$ (right) for different choices of the $\varepsilon_{c}^{\Lambda}$ parameter for $c \rightarrow \Lambda_{c}$ transition in the Peterson fragmentation function.

\section{Conclusions}

In one of our recent papers we demonstrated that the production of high-energy neutrinos is related to very high $p p$ collision energies (even larger than at the LHC) and rather large $x_{F}$ (not accessible at the LHC). Do we know mechanisms of $D$ meson production in these regions?

Here we have presented and discussed briefly some results on asymmetry in the production of $D^{+}$and $D^{-}$[6] as well as $D_{s}^{+} D_{s}^{-}$mesons [12] observed recently by the $\mathrm{LHCb}$ collaboration $[4,5]$. Here we have discussed a scenario in which subleading (unfavored) fragmentation $q / \bar{q} \rightarrow D^{ \pm}$is responsible for the asymmetry. In the case of $D^{+} D^{-}$asymmetry it is quark-antiquark asymmetry in the nucleon which is responsible for the effect. Adjusting the corresponding quark/antiquark fragmentation probability we were able to describe the corresponding asymmetry. This has dramatic consequences for low collision energies. We predicted huge asymmetries for RHIC and even larger for lower energies. We hope this will be verified in future by planned or possible to perform experiments. It is not yet checked what are consequences of the subleading fragmentation for high-energy neutrino production.

The asymmetry in the production of $D_{s}^{+}$and $D_{s}^{-}$mesons is a bit more subtle. Here we have $\bar{s} \rightarrow D_{s}^{+}$and $s \rightarrow D_{s}^{-}$subleading fragmentations. The asymmetry of $D_{s}^{+}$and $D_{s}^{-}$production is possible provided there is $s(x) \neq \bar{s}(x)$. Recently we have used one of the CTEQ parton distributions from the fit which allows such a $s-\bar{s}$ asymmetry in longitudinal momentum 
fraction. Our approach gives then correct sign of the asymmetry and it was possible to find corresponding transition probability to roughly describe the LHCb data. This procedure was used to calculate flux of $\tau$ neutrinos produced in the atmosphere. A significant enhancement was suggested. There are first trials to identify $\tau$ neutrinos with the help of IceCube aparatus [13].

Finally we have discussed production of $\Lambda_{c}$ baryons within independent-parton fragmentation picture. It was demonstrated that such a picture is insufficient to consistently describe new LHC data. Especially for midrapidities (ALICE experiment) one observes a significant enhancement compared to the results with corresponding fragmentation probabilities $c / \bar{c} \rightarrow \Lambda_{c}$ obtained from $e^{+} e^{-}$collisions as well as for lower proton-proton collision energies. This strongly suggest a new mechanism. Quark recombination is a good candidate.

\section{Acknowledgments}

This study was partially supported by the Polish National Science Center grant DEC2014/15/B/ST2/02528 and by the Center for Innovation and Transfer of Natural Sciences and Engineering Knowledge in Rzeszów.

\section{References}

[1] R. Maciula and A. Szczurek, Phys. Rev. D 87, no. 9, 094022 (2013).

[2] A. van Hameren, R. Maciula and A. Szczurek, "Single-parton scattering versus doubleparton scattering in the production of two $c \bar{c}$ pairs and charmed meson correlations at the LHC", Phys. Rev. D89 (2014) 094019.

[3] C. Peterson, D. Schlatter, I. Schmitt and P. M. Zerwas, Phys. Rev. D 27, 105 (1983).

[4] R. Aaij et al. [LHCb Collaboration], Phys. Lett. B 718, 902 (2013).

[5] R. Aaij et al. [LHCb Collaboration], J. High Energy Phys. 08, 008 (2018).

[6] R. Maciuła and A. Szczurek, "D meson production asymmetry, unfavored fragmentation, and consequences for prompt atmospheric neutrino production", Phys. Rev. D 97 (2018) 074001.

[7] H. Holtmann, A. Szczurek and J. Speth, Nucl. Phys. A569 (1996) 631.

[8] H. L. Lai, P. M. Nadolsky, J. Pumplin, D. Stump, W. K. Tung and C.-P. Yuan, J. High Energy Phys. 04, 089 (2007).

[9] V. Goncalves, R. Maciuła, R. Pasechnik and A. Szczurek, "Mapping the dominant regions of the phase space associated with $c \bar{c}$ production relevant for the prompt atmospheric neutrino flux", Phys. Rev. D96 (2017) 094026.

[10] M. G. Aartsen et al. [IceCube Collaboration], Science 342, 1242856 (2013).

[11] M. G. Aartsen et al. [IceCube Collaboration], Astrophys. J. 833, no 1,3 (2016).

[12] V. Goncalves, R. Maciuła and A. Szczurek, "From $D_{s}^{ \pm}$production asymmetry at the LHC to prompt $v_{\tau}$ at IceCube", arXiv:1809.05424.

[13] M. G. Aartsen et al. [IceCube Collaboration], Phys. Rev. D 93, no. 2, 022001 (2016); M. Usner [IceCube Collaboration], PoS ICRC 2017, 974 (2018).

[14] R. Maciuła and A. Szczurek, "Production of $\Lambda_{c}$ baryons at the LHC within $k_{t^{-}}$ factorization approach and independent parton fragmentation",

Phys. Rev. D98 (2018) 014016. 